Hepatology, University College London, London, UK; ${ }^{4}$ Department of Surgery, Imperial College London, London, UK; ${ }^{5}$ Department of Oncology, Imperial College London, London, UK; ' SStatistical Genetics Unit, Royal School of Hygiene and Tropical Medicine, London, UK

Introduction Sporadic cholangiocarcinoma (CC) occurs insubjects with no known risk factor for the disease. Its pathogenesis is likely to involve complex interaction of environmental and human factors, including genetic variation. Polymorphisms in natural killer cell receptor protein G2D (NKG2D) have been associated CC in subjects with primary sclerosing cholangitis (PSC). In a recent study, comparing 49 subjects with CC to 316 subjects with PSC and no CC, two single nucleotide polymorphisms (SNPs) in NKG2D were associated with an increased risk of CC: rs11053781 (OR 2.08, p value 0.011) and rs2617167 (OR 2.32, $p$ value 0.0020 ). We aimed to test whether variation in NKG2D also modifies susceptibility to sporadic CC.

Methods DNA was collected from 172 subjects with sporadic CC and a matched control cohort of 256. Haploview v 4.2 was used to select SNPs capturing common genetic variation around the NKG2D gene (MAF $>0.05$, pair-wise comparisons). This identified 7 SNPs to be genotyped, including rs11053781 and rs2617167. Genotyping was performed with a PCR based, robotic system. Hardy-Weinberg equilibrium testing, Armitage trend testing and haplotype frequency comparisons were undertaken. Correction for multiple testing was performed using a permutation method.

Results All genotypes were in Hardy-Weinberg equilibrium. None of the individual SNPs were significantly associated with altered susceptibility to CC. Haplotype analysis revealed that no haplotypes significantly modified risk of CC.

Conclusion This is the first study to examine NKG2D polymorphisms in sporadic CC. The study was well powered. We found no relationship between variation in NKG2D and susceptibility to CC, in contrast to prior findings in PSC patients with CC. This finding may reflect an important difference between the pathogenesis of sporadic CC and that of PSC related CC. It might point to a false-positive result in the prior study of PSC related CC, but it was well executed and produced strong, positive results. This could be elucidated in an additional candidate-gene validation study. However, with increasing availability and affordability, a genome wide association study (GWAS) may prove a more efficient method for further exploring genetic factors in cholangiocarcinoma.

Competing interests None.

Keywords cancer, cholangiocarcinoma, genetics, NKG2D, SNP.

\title{
PTU-120 $\downarrow$ GENETIC VARIATION IN NATURAL KILLER CELL RECEPTOR PROTEIN G2D DOES NOT MODIFY SUSCEPTIBILITY TO SPORADIC CHOLANGIOCARCINOMA
}

